\title{
CAM and reward for wellness
}

\begin{abstract}
CAM strategies are at the same time ancient healing strategy systems, since ayurveda is 5000 years old and Traditional Chinese Medicine with acupuncture is 3000years old, so they are proved to be effective and safe, .... and they are also a new care approach, since occidental world still asks if they are really scientifical despite the evidence, the millennary effectiveness, the thousands of studies and researches, and the fact that people are using them more and more.
\end{abstract}

CAM is called alternative in comparison to the official therapies systems, such as drugs and surgery. Antonovsky starting from 1979 underlines how, in a correct salutogenetic approach, to create health we should at first rule the lifestyle, manage a good diet (nutrition and physical activity), then proceed toward light caring treatments, passing then to acupuncture and such kind of medicine methods, and only in late stages recur to artificial molecules or even more.

It's simple to understand that, as World Health Organization declares "health is a state of complete physical, mental and social well-being and not merely the absence of disease or infirmity", ${ }^{1}$ so from that it derives this observation: relieving symptoms in some ways doesn't correspond to creating wellness. Reaching a well-being global condition is an autonomous process that each person can live, thanks to therapists and all those who want to participate with an active and positive role into others lives.

CAM are indeed lots of healing systems: Traditional Chinese Medicine (acupuncture, chinese fitotherapy, tui na massage, qi-gong, dietetic), ayurveda (yoga, mantra, use of spices and dietetic culture, therapeutical massages); but also integrated therapies, that are: diet, from the greek world síaı $\alpha$ that means lifestyle, particularly nutrition and physical activity, mindfullness and relaxation techniques (from mental training to any oriental zen form of meditation), and even simply understanding that affective life has a main role into our daily routine, becoming of course able to apply this need to our daily habits.

The latest studies focus on reward system, the brain pathways and nuclei involved in the perception and feeling of positive signals and emotions, related to our main needs and istincts, such as food, sex, reproduction, parental behaviours. It is known that when medial paths prevale we have a good mood, positivity, wellness and pleasure perceptions. But in case of stress conditions dopamine is converted into cathecolamines and lateral ways are prevalent, with a global stressful mood and all the organic consequences that derive from that: Mayo Clinic on its site ${ }^{2}$ publishes that stress is strictly related with all the most diffused diseases, such as cardiopathies, gastrointestinal dysfunctions (these two can also be called dysautonomias), allergies and autoimmune disorders, reumatologic diseases, headache/ migraine, insomnia, and all the mood and sex dysfunctions. So it's important to prevent stress, managing it creating wellness daily habits and situations.

In this article I talk about the characteristics and functions of all main CAM (as I said: nutritional and fitness suggestions, acupuncture, ayurveda, mind relaxation tips, and affective life approaches), underlining their neuroscientific and global mechanisms of action: acting on reward system as well, they are able not only to relieve stress, symptoms and pain, but they also can improve in great ways our well-being global condition, and with the wellness feeling that derives from that: we are stronger (getting the so called "resilience", the ability to overcome obstacles), able to face in the better and most positive ways our life, managing it how we want.

Keywords: reward, wellness, health, CAM, stress, acupuncture, ayurveda, diet, nutrition, fitness, mental training, epigenetic, affective life, lifestyle, right to health, prevention, longevity
Volume I Issue 6 - 2015

\section{Dora Dragoni}

Nutritionist, Acupuncture fellow at AMAB, Italy.

Correspondence: Dora Dragoni, Medical Doctor, Nutrionist, personal Trainer, Acupuncture Fellow at AMAB, Italy, Email doradragoni@hotmail.com

Received: October 21, 2015 | Published: October 26, 2015

\section{Introduction}

CAM, complementary and alternative medicines are more and more integrated in our health services, since they not only have an ancient and strong tradition, but they also are proving to be effective and well tolerated by people, they have a good cost-effect ratio, and as I will explain they also base their caring and healing system on natural mechanisms of action, so to impact on our health in a double way ${ }^{3}$ on one hand they are able to relieve light and medium symptoms fastly, on the other hand they also lead to a global wellness condition.

So we have to see these kinds of medical system in a positive and optimistic way, and this can be the good start to approach to any situation. CAM are also real living system, since they consider lifestyle as the main element of our life indeed. Actually we are genes and environment, and lots of studies are underlining that maybe 
environment has more than the past 50\% impact, maybe even $80 \%$ (Belsky): this thanks to epigenetic, the ensemble of mechanisms that act and explain why living in some way is better for our genes, that determine who we are.

I will examine what we can consider CAM, and CAM are becoming any multistrategies approach acting on our lifestyle, then I will talk about reward system, and I will explain their relationships.

\section{CAM}

We can consider CAM any multistrategies approach acting on our lifestyle. They can be named "CAM" in a theoretical opposition to pharmachological drugs, but it is also true that there is the possibility to integrate CAM to drugs, in case CAM are not sufficient to improve people's conditions.

Antonovsky, expert in social medicine, in 1979 started to talk about "salutogenesis", the global theory according to which we are in a continous dynamic equilibrium between health and some kind of disease conditions: in his opinion it is important to pay attention to these stages, in order to recur to simpler and more natural healing systems, such as nutrition, physical activity, therapeutical massages, or even acupuncture, so to reduce the use of drugs and more invasive treatments that are hypothetical sign of more advanced diseases grades.

\section{All CAM strategies consider as basis the body-mind unity.}

\section{Acupuncture}

Acupuncture is part of Traditional Chinese Medicine, and it's typical of all East World, starting from more than 3000 years ago. Acupuncture is based on some ideas. ${ }^{4-7}$

Yin 陰and yang 陽 - synthesized in the TAO 道 symbol (= the path); yin is the cold element, the dark side of a hill, corresponding to night, to feminine element, to sleep, xué-blood, to chronical illness; yang is the hot element, the sun side of a hill, it is related to day, to masculine element, to head, and acut illness

The 5 elements - wood corresponding to liver and gall bladder, the yin organ and yang bowel, fire that is heart and small intestine, earth corresponds to spleen and stomach, metal that is lung and large intestine, water that corresponds to kidney and bladder

Jing luo, the meridians - our body is crossed by 12 main channels linking organs with long skin small areas, 8 extraordinary meridians, then muscle-tendon channels involving mainly muscles, and luo channels, surrounding the main channels

Acupoints - 360 small areas along the superficial meridians; modern science demostrated that they are quite often at a nervousvascular bundle, and this can explain the lots of reflexes that they can trigger

Ba gang, 8 diagnostic rules - yin vs yang, empty vs fullness, surface vs depth, cold vs warmth/hot

3 Treasures - qi, the energy; shen, the soul(s); jing, the reproductive and vital essence

De qi sensation - aching, numbness, tingling, warmth; this phenomenon is physiological and positively related with the good effect of the acupuncture session.
Traditional Chinese Medicine is also:

a. Dietetic approach

b. Phythotherapy

c. Tui na massage, particularly for children

d. Qi-gong, the physical exercise to have outdoor at the open air, to get from the sun energy and positive effects

e. We can also consider the zen meditation practices and lifestyles, as part of TCM

Oriental medicines are also, amongst others aspects:

I. Sho Ni Shin, the japanese pediatric approach, derived from acupuncture and Tu ina

II. Koryo Hand Therapy, a sort of Su Jok - all these microsistems strategies apply acupuncture to one specific body district, in this case the hand; seen that for example the ear is kidney-foetus shaped and so we can relate all organs to it. The acupoints can be stimulated with:

\section{Metal alloy needles}

II. Silver or gold needles, good for a strong tonification

III. Patches with: vaccaria seeds, or magnets, or noble metal alloys

IV. Massage....and according to the disease we can choose distal points to dissipate heat, and stimulating the needles in some particular ways, or proximal points to tonify qi in case of loss and deficit of energy, acting on the needles in different ways.

I will later talk about the effect of acupuncture from an occidentalneuroscientific point of view.

What I want to underline is that, even if chineses already understood that our life is determined by our parents according to what we inherit from them, they also focused a lot on lifestyle ideas useful to improve our quality and quantity of life, a common trait to the more ancient ayurveda traditions.

\section{Ayurveda}

Ayurveda has its roots in India more or less 5000 years ago. The world "ayurveda" means "science of life", infact it is related to all the life theories having as goal longevity and also improving people's quality of life.

The main ayurveda basis is written in two ancient sanscirt texts: Hatha Yoga Pradipika and Shiva Samhita. The basis of those theories and practices are ${ }^{8}$

- Chakra considerations - for indian traditions our body-mind unity has 7 chakras, that we can relate to modern PNEI functions

i. Mulahara - in the perineum, related to sexuality

ii. Svadhisthana - corresponding to genitals - related to relationships and every form of addiction

iii. Manipura - at navel level - related with power, fear and introversion

iv. Anahta - in the heart - related to love and affections 
v. Vishuddi - in the throat, related with communication, and will

vi. Ajna - at the center of brain, and between the eyes (the chinese YIN TANG, the third eye for all oriental cultures), it represents equilibrium and inner guidance

vii. Sahasrara - on the top of the head or even some centimeters above, it is related with spiritual evolution and consciousness, the more elevated human chakra

As I said before most of chakra can be related with PNEI actions, with parasympathetic plexus and endocrinological functions

i. Dietetic suggestions - where the use of spices is a main element

ii. Meditation - mantra meditation formulas, like the famous Om (devanāgari: ॐँ$)$

iii. Body exercises - mainly yoga, based on asana (आसन) the positions to strenghten our body and mind characteristics - yoga has diffused in occidental world for some decades ago and lots of gyms introduced yoga courses for all ages, from children to adults to elderly people

\section{Other CAMs}

With CAM we can also considered occidental integrated therapies, such as:

Diet - the world " $\delta i ́ \alpha ı \tau$ " in ancient greek means "lifestyle" and modern theories include in this mainly:

Nutrition ${ }^{9,10}$ - mediterrenean diet in 2010 has been declared "intangible cultural heritage for humanity" by Unesco - it is based on: non refined complex carbohydrates $<55 \%$ more times a day (they have lower glycemic index), 5 portions of vegetables and fruit rich in fiber and vitamins, white meat, fish (rich in omega-3) and fresh cheese and eggs as proteic intake, dry fruits and nuts (containing omega-6), adding some mediterrenean herbs (as basel, parsel, rosemary, chives, sage, thyme) and reduction of sweet foods; other than at least $2 \mathrm{~L}$ of water per day; it can be very impactful, great for health and wellness - but mediterrenean diet, studied by the american Ancel Keys, who noticed the good quality and longevity of mediterrenean people, is also mediterrenean lifestyle, where sunbathing when possible is always a must

Physical activity - WHO suggested more or less 60minutes per day of light-medium fitness exercises, according to age we can choose: walking, bike riding, or aerobical-anaerobical activities... working out as we prefer according to our habits and goals

Mindfulness and relaxation techniques ${ }^{11}$ lifestyle is also mind and relax, I can suggest mental training techniques: they start from the sport field, so to help an athlete to get the better degree of relaxation before a match, and also to concentrate focusing the better behaviours and athetlic feats to have, but we can apply autogenic training or other visualization strategies to any condition in which we want to reduce and relieve stress in order to get to good mental and emotive pattern all this is typical of positive psychology, the modern way of approach in psychology born in the 1990s and related to Maslow's theories of the $1950 \mathrm{~s}-1960 \mathrm{~s}$, starting from identify the main needs, corresponding to our instincts (physiological - food, sex, sleep; safety - health; love and belonging - sexual intimacy and family creation; esteem - esteem and respect; and self actualization - morality and creativity)
Affective life ${ }^{12,13}$ emotions, love, sexual and reproductive behaviours are part of us since they permit not only a daily body and mind health, but they also are the main basis for our wellness conditions and they assure species continuation - in lack of these we obviously perceive physical pain (as UCLA demostrated in a recent study) - they correspond to material needs, since if they are present we have the right amount of positive molecules (produced and released by thinking to the beloved person, communicating with him/her, sharing daily life with our partner, and also in kissing and physical contacts, and of course mainly produced in orgasm condition) such as hormones, beta-endorfines, dopamine and serotonine, and oxytocin, related to parental behaviour too. In lack of these we have withdrawal symptoms, that are both at psychical and physical level, till to real "somatizations" due to that, with at last stages really organic symptoms. Fajrajzen explained in a 1960s text.$^{14}$ about abstinence how many symptoms and illness considered psychological or even organic are simply due to inadequate affective life, and helping the person to reach a satisfying love life means in most cases to relieve symptoms and getting an health and wellness long lasting life. So it is important that anyone lives her and his own right to have a love life starting from when this is a need, even when these centers become tonically and phasically active according to our human natural essence, that is during adolescence teenage years, personally and freely choosing according to her and his preferences and in the best way to rule a satisfying and complete life in wellness condition.

\section{Reward system}

Reward system is characterized by the brain dopaminergic and serotininergic pathways related to global body-mind health. As I said World Health Organization declared health "as a state of complete physical, mental and social well-being and not merely the absence of disease or infirmity".

Even Mayo Clinic ${ }^{2}$ has focused the attention on the correlation between stress and many common and diffused diseases, such as: headache, gastric symptoms, some chest and heart disfunctions (called also "dysautonomia"), allergies and autoimmune dysfunctions, the trend to get colds and flus, psychological disregulations, being often angry and dissatisfied, have an unsatisfactory affective and sexual life. We can understand how stress and health are related thanks to reward pathway analysis ${ }^{15,16}$ Infact it is composed by two main pathways: the medial related to wellness condition, and the lateral characterized by stress situations.

The main center is VTA, ventral tegmental area, in ventral midbrain: it is made by dopaminergic (and also serotoninergic) neurons projecting to lots of areas:

\section{a. - Thalamus}

b. - Amygdala - the big bilateral nucleus of fear and pleasure

c. - Nucleus accumbens - in which good perceptions take place

d. - Hypothalamus - that, with pituitary gland, regulates our instincts, thanks to hormone secretion

e. - And cortical areas, such as lymbic system, place of emotions, and pre+frontal areas, where behaviours are elaborated.

f. In basic normal conditions we have the prevalence of medial pathways, wellness perceptions and health of body and mind. If stress stimuli impacts on this system dopamine starts to 
decrease, it is transformed into cathecolamines due to an enzime (dopamine-beta-hydroxilase) and so we pass from some hypotalamic productions to others (from gonadotropine prevalent production to $\mathrm{CRH}-\mathrm{ACTH}$-cortisole, the stress axis; this explains also the increase of infertility in occidental world synthetically: fertility is related to lower stress levels and higher rates of regular sexual couple life).

Amydgala starts elaborating fear signals and the risk is that chronical conditions of lateral reward, where medial good and pleasant stimuli are not a daily lived habit, could lead to chronic stress impacting on both mind and on all the main organs as I said before.

\section{Correlations and multi strategies}

So we clearly understand how it is unuseful to act on specifical symptoms, since all ar related to the effect of stress and if we don't act on reward system correctly it is a "dog chasing his tail". For chineses this was already known ${ }^{6,7}$ they distinguish between BEN the root and $\mathrm{BIAO}$ the manifestation. So it is fundamental to prevent and to act on ben, if we pretend to get improvements of biao.

I make a short consideration about the difference with the placebo effect, that too many consider very similar to some CAM effects. CAM effects are more and more studied and they are proved by lots of scientifical studies. In these cases positive effects on reward are objective. On the contary placebo effect is also true and also based on expectancy, but is related to personal subjective experiences and it is usually weaker. I briefly will explain how leading a correct lifestyle, characterized of the fulfillment of all our needs, is the basis to evitate stress conditions that takes place at first when we don't live as we should for who we are, since we shift medial impulses to lateral paths, those of stress and disease.

I will do some examples based on scientific and clinical studies. Before I want to remember that analyzing as I said according to PNEI and to the relationships to orthosympathetic and parasympathetic branches and outflows.${ }^{17}$, it is important to understand that every basal or acute stress condition needs to be balanced with a basal or increased parasympathetical discharge: an example is stress gastric ulcer, due to such a strong amount of stress that gastric mucosa gets injured by increased acid secretions. Of course, as also chakra knowledge teaches, all parasympathetic should be in balance: eyes, thoracic and heart ones, gastrointestinal and sacral. If one of them prevale, symptoms start. Even every drug should keep this into consideration, so to evitate overdoses effects and consequent diseases deriving from that, the opposite to what had to be set, the healing and wellness conditions.

Talking about acupuncture, I can shortly remember some recent studies:

Almeida in his book ${ }^{18}$ describes how stress -> CHR -> POMC (pro-opium-melano-cortin, from which melanine and opioids) -> both ACTH-cortisol (the adrenal gland molecule typical of stress) AND opioids - so this means that stress leads to opioids production. But opioids, according to Johnson, ${ }^{19}$ lead to a relative reduction of GABA (the inhibitory molecule) in midbrain, so that we don't live the negative effects of it but normal dopamine and serotonine levels can be restored

Dopamine and opioids are always linked, since

(a) dopamine is precursor of endgoneous morphine. ${ }^{20,21}$ (b) opioids reduce the pain experience, reducing the signals of pain matrix (concept developed by Melzack), the neural webs elaborating pain signals in our brain (even affective and social pain ones), and dopamine is positively related with medial reward signals, essential basis for an health and wellness condition

In each case Yoshimoto describes ${ }^{22}$ how acupuncture leads to increase of serotonine more than dopamine, assuring in this way to have the correct balance of subcortex-cortex equilibrium. So we understand how acupuncture not only has nociceptive effects great to relieve pain, but also impact in a positive way on our global health and wellness equilibrium, assuring long term improvements, free of therapies. Reward is also related with neurogenesis: acupuncture has this effect and Chiu in 2015 demostrated how L-DOPA is also neurogenetic. ${ }^{23}$

To get real free of therapies conditions, we should act strongly on lifestyle. I recently underlined the relationships between food and reward ${ }^{10}$ and also how fitness is related with wellness perceptions: it reduces stress as well, and beta-endorfin as endogenous opioids molecule are basis for long wellness conditions. It also strenghten our body and mind patterns. And thanks to NO, nitric oxyd, as acupuncture has a role of organ protection..$^{12}$ And of course, as I before explained, a satisfying affective life with our partner is great part of ourselves. ${ }^{12,13}$ Love life is part of us and we should have the possibility to live our present and future with a partner who shares with us trust and belief. Regular active love life is related with better health according to all researches and also to longevity, better in quantity and quality (free of degenerative diseases). A recent study (2015) by Southwest University and University of Science and Technology of China, collaborating with Mount Sinai School of Medicine of NY, guided by Xiaochu Zang, ${ }^{24}$ evidenced how the more reward system is activated (MRI imaging) the more quality and lasting of a love relationship between two partners are good.

\section{Conclusive perspectives}

In conclusion I would say that CAM therapies can be considered not only traditional oriental healing systems, but all the integrated multistrategies based on natural rewards (concepts elaborated also by Esch and Stefano, ${ }^{20,21}$ and P.S. Grigson).

So it is important to have a correct lifestyle, being conscious of how needs and on how living them in our daily routine: in case of organic dysfunctions we could recur to any kind of therapy, but all agree on the fact that prevention is the main step to take. It is also important to have guaranteed both rights to health and to affective life (even World Association for Sexual Health constantly revises his Declaration, in which some of these rights are contained), that as I explained are so strictly related.

Eating in the right way, even respecting sustainability and preferring organic and local food so to contribute in saving at the same time the Earth equilibrium, having fitness time every day, and living love with our partner are the keys to better life, health and wellness.

\section{Acknowledgement}

A special thanks to Fatih Divrak.

\section{Conflicts of interest}

The authors declare that there are no conflicts of interest. 


\section{Funding}

None.

\section{References}

1. www.who.int

2. www.mayoclinic.org

3. Takakura N, Takayama M, Kawase A, et al. Double-blind acupuncture needle:a potential tool to investigate the nature of pain and pleasure. ISRN Pain. 2013;2013.pii: 825751.

4. www.agopuntura-fisa.it

5. amabonline.it

6. Maciocia, The Foundations of Chinese Medicine. Masson.

7. Sotte, Minelli E, Giovanardi C, et al. Fundamentals of acupuncture and Chinese medicine. USA: CEA; 2006. p. 744.

8. Da Re Lombardi G, guida pratica. Chakra.

9. Oxford handbook of nutrition and dietetic.

10. Dragoni D. Diet Health and Wellness. Journal of Nutritional Health and Food Engineering. 2015;2(5):00073.

11. Brugnoli, Tecniche di Mental Training nello sport, Edizioni red!

12. Volkow ND, Wang GJ, Baler RD. Reward dopamine and the control of food intake:implications for obesity. Trends Cogn Sci. 2011;15(1):37-46.

13. Fisher, Why we love?

14. Fajrajzen, L'astinenza sessuale Manual de medicinas complementares. OCEANO.
15. Dreher Tremblay. Handbook of reward and decision making. 1st edn, USA: Academic Press; 2009. p. 526.

16. Dragoni D. (2012; 2015) REWARD7. IlMioLibro (italian+english in front).

17. Almeida. Neurobiology of Opioids. The Neurobiology of Acupuncture. International Review of Neurobiology, handbook of stress science: biology, psychology and health. $1^{\text {st }}$ edn. USA: Academic Press Contrada; 2013. p. 360.

18. Johnson SW, North RA. Opioids excite dopamine interneurons by hyperpolarization of local interneurons. J Neurosci. 1992;12(2):483488.

19. Esch T, Stefano GB. Endogenous reward mechanisms and their importance in stress reduction, exercise and the brain. Arch Med Sci. 2010;6(3):447-455.

20. Esch T, Stefano GB. The neurobiology of pleasure, reward processes, addiction and their health implications. Neuro Endocrinol Lett. 2004;25(4):235-251.

21. Yoshimoto K, Fukuda F, Hori M, et al. Acupuncture stimulates the release of serotonin but not dopamine in the rat nucleus accumbens. Tohoku J Exp Med. 2006;208(4):321-326.

22. Chiu WH, Depboylu C, Hermanns G, et al. Long-term treatment with L-DOPA or pramipexole affects adult neurogenesis and corresponding non-motor behavior in a mouse model of Parkinson's disease. Neuropharmacology. 2015;95:367-376.

23. Song H, Zou Z, Kou J, et al. Love-related changes in the brain:a restingstate functional magnetic resonance imaging study. Front Hum Neurosci. 2015;9:71. 